\begin{tabular}{|c|c|}
\hline Title & Simulation A nalysis of Tilted Polyhedron-Shaped Thermoelectric Elements \\
\hline Author(s) & Meng, X iangning; Suzuki, Ryosuke 0. \\
\hline Citation & $\begin{array}{l}\text { Journal of electronic materials, 44(6), 1469-1476 } \\
\text { https://doi.org/10.1007/s11664 014-34185 }\end{array}$ \\
\hline Issue Date & $2015-06$ \\
\hline Doc URL & http:/hdl.handle.net/2115/61966 \\
\hline Rights & The final publication is avail able at Springer via http://dx.doi.org/10.1007/s11664-014-3418.5 \\
\hline Type & article (author version) \\
\hline File Information & Suzuki-JEM.pdf \\
\hline
\end{tabular}

Instructions for use 


\section{Simulation analysis of tilted polyhedron-shaped thermoelectric elements}

Xiangning Meng ${ }^{1,2 *}$ and Ryosuke O. Suzuki ${ }^{1,3}$

Contact e-mails: mengxn@eng.hokudai.ac.jp and rsuzuki@eng.hokudai.ac.jp

1. Faculty of Engineering, Hokkaido University, Sapporo, Hokkaido 060-8628, Japan

2. School of Materials and Metallurgy, Northeastern University, Shenyang 110819, China

3. Japan Science and Technology Agency, JST, Tokyo, Japan 


\section{Abstract}

The generation of thermoelectricity is considered as a promising approach to harness the waste heat generated in industries, automobiles, gas fields, and other man-made processes. The waste heat can be converted to electricity via a thermoelectric (TE) generator. In this light, the generator performance depends on the geometric configuration of its constituent elements as well as their material properties. Our previous work reported TE behaviors for modules consisting of parallelogram-shaped elements because elements with tilted laminate structures provide increased mechanical stability and efficient heat transferring ability from the hot surface to the cold surface. Here, we study TE elements in the shape of a polyhedron that is obtained by mechanically truncating the edges of a parallelogram element in order to further enhance the generator performance and reduce TE material usage. The TE performance of the modules consisting of these polyhedron elements is numerically simulated by using the finite-volume method. The output power, voltage, and current of the polyhedral TE module are greater than those of the parallelogram-element module. The polyhedron shape positively affects heat transfer and the flow of electric charges in the light of increasing the efficiency of conversion from heat to electricity. By varying the shape of the truncated portions, we determine the optimal shape that enables homogeneous heat flux distribution and slow diffusion of thermal energy to obtain the better efficiency of conversion of heat into electricity. We believe that the findings of our study can significantly contribute to the designing policy in TE generation.

Key words: Thermoelectric generation; Thermoelectric module; Polyhedron elements; Finite-element analysis 


\section{Introduction}

The heat at a junction of two different materials can be directly converted into electricity by using a thermoelectric (TE) generator, which is based on the Seebeck effect. This method of generating electricity has many advantages. Inexhaustible resources and low-grade energy, such as renewable solar heat and unrecovered waste heat, can be utilized for this method. Further, thermoelectric generators (TEGs) do not utilize chemical reactions or mechanical moving parts in their operation. Consequently, TEGs are noiseless, pollution-free and can even be operated in isolated locations. However, the low energy conversion efficiency of a TEG is its primary deficiency $^{[1,2]}$, even though several advancements have been made in terms of fabricating more efficient TE materials ${ }^{[3,4]}$. Other factors to maximize TEG efficiency include optimal design of the TEG system because the heat harvest and output power can be significantly enhanced by optimizing the configurations and geometries of TE panels, modules, and elements ${ }^{[5,6]}$.

The preferential flow of current in a given three-dimensional (3D) TE system can be studied via analysis of the TE modules consisting of $p$ - and $n$-type elements, which are connected thermally in parallel and electrically in series $^{[7]}$. This current path is formed spontaneously, causing electric charges to flow through the shortest path, and regions with high current density are formed at the diagonal corners of TE elements. Recently, it has been reported that the TE performance of a given system can be enhanced by using a tubular TE device consisting of a tilted laminate structure, which provides good mechanical stability and allows direct and efficient heat transfer from fluid heat sources ${ }^{[8,9]}$. In this design, TE elements are tilted against the direction of the primary current stream to minimize the volume occupation of areas with low current density. In this light, the TE performance of such a system has further been simulated for modules comprising tilted elements ${ }^{[10]}$. The results for isotropic TE materials show that the tilted element enables primary current flows at higher densities; however, the presence of the tilted element also creates prominent regions with low current densities, thereby causing an obvious 
inhomogeneous current density over the entire element. Consequently, a new conceptual design of polyhedral-shaped TE elements has been proposed by cutting away identical-sized triangular prisms from the two long-diagonal edges of the previously used parallelogram element terminals ${ }^{[10]}$. The two corner sections of the tilted elements are truncated away at an angle $\theta$ to the horizontal (hereafter defined as the truncating angle), as depicted in Figure 1 (a). The elements themselves are tilted at an angle $\alpha$, as also illustrated in the figure. This design eliminates regions with low-density currents, and further, it reduces thermal energy and material wastage by suppressing the electric and thermal resistances of the TE module. The truncated portions are instead filled by appropriately shaped electrode material with a higher conductivity. Figure 1 (b) shows the example of such a $p$ - $n$ pair. In this study, we investigate the performance of such polyhedral-shaped TE elements.

The primary purpose of this study is to investigate the influence of the polyhedron geometry on the TE performance. The relationship between the truncating angle $\theta$ and the TE performance in the module consisting of these polyhedron elements is examined, and subsequently, we present the optimal polyhedron design for these tilted elements. The removal of the two identical prism sections from the two corners of the parallelogram is implemented with the element itself being tilted at an angle $\alpha$ (for example, $\alpha=45^{\circ}$ ). For simplicity, the guidelines for the truncation of the two corners are set based on the following assumptions: (1) the total volume of the truncated-off portions is accounted as one-fifth that of the entire element, (2) a truncating angle of $67.5^{\circ}$ ensures that the cross-section of the prisms form an isosceles triangle with an apex angle of $45^{\circ}$, and (3) truncating angles of $55^{\circ}$ and $80^{\circ}$ are taken as the angles sandwiching the central angle of $67.5^{\circ}$. The designs of the tilted polyhedron elements thus formed are listed in Table 1. The original tilted parallelogram element is considered as an extreme case of polyhedron geometry with $\theta=0$.

\section{Modeling}




\subsection{Basic Conditions}

Finite-element analysis is conducted on modules consisting of 18 pairs of $p$ - $n$ elements in series to examine the 3D TE effects. The common geometries for all the modules are listed in Table 2. The properties of the materials used in the module ${ }^{[11]}$ are listed in Table 3. For simplicity, the following assumptions are made in formulating the entity models: (1) the entire TE system is adiabatic with respect to the external environment, (2) all the parts of the module are perfectly bound to each other, and (3) the properties of the materials used are temperature-independent and isotropic in the light of understanding the underlying fundamental macroscopic TE phenomena. In our study, the surface temperature of the hot and cold sources was set to be homogenous over the entire module. The temperature of the cold surface, $T_{2}$, was fixed at $300 \mathrm{~K}$ to represent the ambient temperature, while the temperature of the hot surface, $T_{1}$, was varied sequentially as $400 \mathrm{~K}, 450 \mathrm{~K}, 500 \mathrm{~K}, 550 \mathrm{~K}$, and $600 \mathrm{~K}$. The macroscopic heat balance in the TE module is illustrated in Figure 2, and this heat balance can be expressed by Equation (1) and Equation (2). The theoretical output power is consequently given by Equation (3).

$$
\begin{gathered}
Q_{1}=Q_{\mathrm{c}}+Q_{\mathrm{P} 1}-\frac{1}{2} Q_{\mathrm{J}} \\
Q_{\mathrm{c}}+Q_{\mathrm{P} 2}+\frac{1}{2} Q_{\mathrm{J}}=Q_{2} \\
P=Q_{\mathrm{P} 1}-Q_{\mathrm{P} 2}-Q_{\mathrm{J}}
\end{gathered}
$$

Here, $Q_{1}$ and $Q_{2}$ denote the heat quantities conducted through the layers of the insulator and electrode, respectively. The parameters $Q_{\mathrm{P} 1}$ and $Q_{\mathrm{P} 2}$ denote the Peltier heat, and these parameters are dependent on the temperature and current. These heat fluxes appear at the interfaces between the electrodes and the TE elements. 
The parameters $T_{\mathrm{h}}$ and $T_{\mathrm{c}}$ denote the surface temperatures in the hot and cold sides of the elements, respectively. The term $Q_{\mathrm{J}}$ denotes the Joule heat following Joule's law, $Q_{\mathrm{c}}$ denotes the total heat conducted through all the TE elements, and $P$ denotes the output power of the TE module.

\subsection{Constitutive Equation}

The internal heat transfer and electric charge transportation are analyzed based on the thermal diffusion and charge transportation equations, respectively, in addition to the TE phenomena. The differential equations for heat conduction at each finite-element volume are solved on the basis of energy conservation. The heat-conduction equation under steady-state conditions is given by Equation (4).

$$
\nabla \cdot(\lambda \Delta T)+\rho|\boldsymbol{J}|^{2}-T \boldsymbol{J} \cdot \nabla S=0
$$

Here, the three terms represent the heat conduction, the Joule heat generated by the current along the finite-element volume, and the heating or cooling generated by the Thomson effect in the given order. The parameters $\lambda, \rho$, and $S$ denote the thermal conductivity, specific electric resistivity, and relative Seebeck coefficient, respectively. The parameter $\boldsymbol{J}$ denotes the current density determined by the electric potential and temperature obtained using Equation (5).

$$
\rho \boldsymbol{J}=-\nabla V-S \nabla T
$$

Here, the voltage due to $\rho \boldsymbol{J}$ is expressed by the change in the electric potential $(\nabla V)$ and the voltage generated from the Seebeck effect $(S \nabla T)$. The differential equation, Equation (6), is derived from Equation (5) by 
applying charge conservation under steady-state conditions.

$$
\nabla \cdot\left(-\frac{1}{\rho} \nabla V\right)=\nabla \cdot\left(-\frac{s}{\rho} \nabla V\right)
$$

The temperature and electric potential distributions are obtained by numerically solving the simultaneous differential equations of Equation (4) and Equation (6) with the finite-volume method using the commercial software package, FLUENT. By referring to the reported codes ${ }^{[12,13]}$, we evaluated the current density via our custom-written C program ${ }^{[7,14]}$, and subsequently, our codes were combined with the conventional functions of FLUENT because this commercial package does not account for this TE phenomena. The final results were obtained after several thousand iterations until sufficient convergence was achieved for each complex calculation of the entire TE process.

\section{Results and Discussion}

\subsection{Performance Comparison}

In general, the voltage and current increase linearly with the temperature difference between the hot and cold sources, $\Delta T=T_{1}-T_{2}$, for all element topologies because the electromotive force acting on the TE module is the sum of the products of the relative Seebeck coefficient $S$ and $\Delta T$ for all the serial connections in the module. The output power $P$ generated by the TE module is proportional to the square of $\Delta T$. The conversion efficiency $\eta$ is defined as the ratio of the output power $P$ to the input heat $Q_{1}$ absorbed at the hot-source surface. The TE performance of the module is significantly enhanced for TE elements shaped in the form of polyhedron when compared with that of tilted parallelogram TE elements. A higher temperature difference $\Delta T$ leads to increased performance. For the same value of $\Delta T$, there is no distinct difference in terms of the voltage and current for each 
TE module consisting of polyhedral elements. However, the voltage and current generated by polyhedron elements are clearly higher than those generated by the parallelogram elements (Case-4 in Figure 3 (a) and (b)). For example, the maximum changes in the voltage and current at $\Delta T=300 \mathrm{~K}$ are about $0.03 \mathrm{~V}$ and $0.13 \mathrm{~A}$, respectively. The utilization of polyhedron elements is more advantageous than the utilization of parallelogram elements. Interestingly, Case-2 in Figure 3 (c) exhibits the lowest input heat among all the cases. This depends on the heat flux distribution and the configuration of the TE module when the surface temperatures of the hot and cold sources are set constant. However, the output power of the module using this design (Case-2) is comparable to those of the other designs, as can be observed from Figure 3 (d). Thus, Case-2 corresponds to the highest efficiency of converting heat energy into electricity.

From Figure 4, we note that the TE performance varies with the truncating angle $\theta$ as it is varied from $55^{\circ}$ (Case-1) to $80^{\circ}$ (Case-3). In this light, we observe that Case-2 corresponds to the minimum voltage, current, input heat, and output power both at $\Delta T=200 \mathrm{~K}$ and $\Delta T=100 \mathrm{~K}$, as delineated by the dashed-line box in Figure 4, and further, the conversion efficiency for this design is maximum. Such TE performance variation is due to inconsistencies in the assembly of module components that have different material properties. The change in the module configuration caused by varying the truncating angle affects the heat transfer, heat flux distribution, and electric charge movement of the entire module, as discussed later.

\subsection{Module Features}

The use of the polyhedron design with any truncating angle positively influences the current density of the TE elements. As shown in Figure 5, low-current-density regions in the parallelogram elements appear at the two small areas close to the terminals lying at either end of the long diagonal, although the polyhedron design leads to a more homogenous distribution in current density than that obtained with parallelogram elements. This is 
because the current preferentially flows through the shortest path available to form a primary current stream, and this path is determined by the configuration of the TE module ${ }^{[7]}$.

As regards the polyhedral elements, the corners lying at either end of the long diagonal of the parallelogram element are truncated to form the polyhedron shape, and electrode plates with a higher electrical conductivity (shaped in the form of the truncated prismatic sections) are affixed such that the current pathway is widened, and consequently, the probability of the electric charges being transported through the entire element increases. Furthermore, the current density on the electrode plate in contact with the $p$-type polyhedron element as regards Case-2 is also homogenously distributed. In essence, these current-density behaviors can be reasonably explained by current seeking the shortest flow path.

The effect of the polyhedron geometry on the current flow can be compared in Figure 5 (a) and (b). The figures show the direction and intensity of current in each control unit by means of arrows. Obviously, the current flows in two different paths of similar lengths in the p-type polyhedron element for Case-2, as shown in Figure 5 (a). The polyhedral shape works in homogenizing the current density. However, almost all the current segments join at the terminal of sole mainstream in the $p$-type parallelogram element in Case-4, as shown in Figure 5 (b). As regards the $n$-type elements, the current mainstream flows along two paths in the polyhedron element and via only one path in the parallelogram element. However, certain regions with very small values of current density exist regardless of the current flow pattern mentioned above.

We note here that the simulation results show that the distributions of the current density in the TE module are roughly similar for Case-1, Case-2, and Case-3. Consequently, the enhanced TE performance for Case-2, as can be observed in Figure 4, can be attributed to the thermal behavior in the TE module. From Figure 6, we note that the temperature distributions in the module largely depend on the material properties of the individual components. The temperature of the electrode plates is maintained equal to the temperatures of the hot or cold 
sources due to its very high thermal conductivity, while significant temperature contours are formed within the TE elements because of their lower thermal conductivity. This strong relationship between the temperature distribution and the thermal conductivity is not affected by the value of the optimal truncating angle (Case-2) because no significant changes are observed in the temperature distribution when the truncating angle is varied from $55^{\circ}$ (Case- 1$)$ to $80^{\circ}$ (Case-3).

The heat fluxes for each of these designs are shown in Figure 7. Most areas of the elements exhibit a homogenously small heat flux due to the lower thermal conductivity of the element and the consequently slower thermal diffusion through the material. The higher thermal conductivity of the electrode plates leads to the formation of a higher heat flux, thereby leading to an inhomogeneous heat distribution because of the irregular geometry of the plates. We note that a higher heat flux clearly emerges at locations close to the terminals of the current mainstream in the elements, thereby indicating that heat is also preferentially transferred through a "fast route" in a manner similar to the current flow. This geometry-dependent route is inevitably affected by the module configuration. Local differences in the heat flux exist in the TE module for all designs. For example, a large amount of heat is spontaneously transferred along the short diagonal of the $p$-type element for Case- $1(\theta=$ $55^{\circ}$ ), and two significant high-flux regions with a maximum heat flux of $1190 \mathrm{~kW} \cdot \mathrm{m}^{-2}$ are formed at both terminals (lying at either end of the short diagonal) of this path, indicated in red in Figure 7 (a). The flux in these regions gradually reduces as the truncating angle increases to $67.5^{\circ}$. The heat flux is more homogenous for this design, and the maximum flux is reduced to $1120 \mathrm{~kW} \cdot \mathrm{m}^{-2}$, as shown in Figure 7 (b). Another short heat-transfer path is created when the truncating angle $\theta$ is increased to $80^{\circ}$, and two new high-flux regions emerge at the edge terminals of the electrode, which are delineated by the black circles in Figure 7 (c). In this case, the maximum heat flux is $1230 \mathrm{~kW} \cdot \mathrm{m}^{-2}$, thereby indicating that the distribution of heat flux is no longer as homogenous as that for Case- $2\left(\theta=67.5^{\circ}\right)$. Further, the maximum heat flux reaches $1400 \mathrm{~kW} \cdot \mathrm{m}^{-2}$ for Case- 4 . 
These high-flux regions are highlighted in both the $p$ - and $n$-type elements in Figure 7 (d).

The minimum and maximum values of the heat flux and current density for all the designs are listed in Table 4.

These minimum and maximum values and their differences reflect the uniformity of heat and charge flow over a constant volume, and thus, they can be used to evaluate the choice of the module configuration. For example, unnecessary heat consumption and excessively low current density are undesirable from the viewpoint of reduction in energy and material wastage. From the results, we note that the minimum values of the heat flux and current density for Case-2 are larger than those of Case-1 and Case-3, and the maximum values in Case-2 are smaller than those of Case-1 and Case-3. That is, the ranges between maximum and minimum values of heat flux and current density are the smallest for Case-2, for which design the heat flux and current density are mostly homogeneous.

Further, we find that rapid heat diffusion occurs in TE module consisting of parallelogram elements, which is not favorable for TE conversion. This rapid heat transfer explains the reason for the poor performance observed for Case-4. However, the rate of heat transfer relatively reduces for Case-2, and as a result, no further heat is absorbed because of the inefficient thermal diffusion. Because of the geometry similarity, small differences exist in the TE performances in terms of voltage, current, and output power for the three designs, Case-1, Case-2, and Case-3, due to the slight difference in the electrical resistance caused by configuration changes, while the difference is significant for the parameter of input heat. The polyhedral-element configuration aids in reducing energy dissipation and enhancing the TE conversion efficiency. In addition, the maximum current density for Case-2 is not significantly different from those of Case-1 and Case-3. This indicates that the module configuration with polyhedron elements with a truncating angle $67.5^{\circ}$ is most suitable among the three designs, and further, electric charges are most homogenously distributed over a fixed space in this configuration. 


\section{Conclusions}

This paper proposed the use of TE elements designed in the shape of polyhedron, which can be realized by mechanically truncating TE material from the edges along a line perpendicular to the long diagonal axis of parallelogram elements, whose morphology has been studied in a previous work. Here, we considered the case wherein two identical triangular prisms were cut away from the long-diagonal terminals of the tilted parallelogram elements. The tilting angle was set to $45^{\circ}$ and the truncating angle was varied as $55^{\circ}, 67.5^{\circ}$, and $80^{\circ}$, which resulted in three element designs. The simulation results for isotropic TE materials showed that the design denoted as Case-2 (tilting angle of $45^{\circ}$ and truncating angle of $67.5^{\circ}$ ) provides optimum TE performance under conditions of fixed temperatures of the hot and cold sources; this design is most promising in terms of practical application. This design is characterized by homogeneity of the heat flux across the elements and the slow diffusion of thermal energy, and hence, it is the optimal design towards achieving a high efficiency of converting heat into electricity. We believe that our findings can significantly contribute to the practical realization of an efficient system to generate thermoelectricity. 


\section{Acknowledgements}

The authors are grateful to financial support from JST-CREST, Research Grant-in-Aid of JSPS (No. 24656574 and 26630490), and JSPS Fellowship. 


\section{References}

1. K. Koumoto, R. Funahashi, E. Guilmeau, Y. Miyazaki, A. Weidenkaff, Y. Wang and C. Wan: J. Am. Ceram. Soc. 96 (2013) 1-23.

2. G. J. Snyder and E. S. Toberer: Nat. Mater. 7 (2008) 105-114.

3. T. Aoki, C. Wan, H. Ishiguro, H. Morimitsu and K. Koumoto: J. Ceram. Soc. Jpn. 119 (2011) 382-385.

4. T. Sakamoto, T. Iida, A. Matsumoto, Y. Honda, T. Nemoto, J. Sato, T. Nakajima, H. Taguchi and Y. Takanashi: J. Electron. Mater. 39 (2010) 1708-1713.

5. A. Z. Sahin and B. S. Yilbas: Energy Conv. Manag. 65 (2013) 26-32.

6. A. Takezawa and M. Kitamura: Int. J. Numer. Methods Eng. 90 (2012) 1363-1392.

7. X. Meng, T. Fujisaka and R. O. Suzuki: J. Electron. Mater. 43 (2014) 1509-1520.

8. K. Takahashi, T. Kanno, A. Sakai, H. Tamaki, H. Kusada and Y. Yamada: Sci. Rep. 3 (2013) 1501.

9. T. Kanno, A. Sakai, K. Takahashi, A. Omote, H. Adachi and Y. Yamada: Appl. Phys. Lett. 101 (2012) 011906.

10. X. Meng, T. Fujisaka, K. O. Ito and R. O. Suzuki: Mater. Trans. 55 (2014) 1219-1225.

11. D. M. Rowe: CRC Handbook of Thermoelectrics, (CRC Press, Florida, 1995) pp. 445-446.

12. M. Chen, L. A. Rosendahl and T. Condra: Int. J. Heat Mass Transf. 54 (2011) 345-355.

13. R. O. Suzuki, Y. Sasaki, T. Fujisaka and M. Chen: J. Electron. Mater. 41 (2012) 1766-1770.

14. T. Fujisaka, H. Sui and R. O. Suzuki: J. Electron. Mater. 42 (2013) 1688-1696. 


\section{Figure captions}

Figure 1 Schematic representation of tilted polyhedron element (a) and p-n pair (b).

Figure 2 Schematic representation of heat balance of TE module.

Figure 3 TE performances of modules using 4 designs: (a) voltage, (b) current, (c) input heat, (d) output power and (e) conversion efficiency.

Figure 4 Performance comparisons of TE modules: (a) voltage, (b) current, (c) input heat, (d) output power and (e) conversion efficiency.

Figure 5 Current densities in TE elements of (a) polyhedron-shaped in Case-2 and (b) parallelogram-shaped in Case-4.

Figure 6 Temperature in TE elements of (a) polyhedron-shaped in Case-2 and (b) parallelogram-shaped in Case-4.

Figure 7 Heat flux in TE elements of polyhedron-shaped in (a) Case-1, (b) Case-2, (c) Case-3 and (d) parallelogram-shaped in Case-4. 
Table captions

Table I Designs of tilted polyhedron elements.

Table II Configuration data of TE module.

Table III Thermal and electric properties of materials ${ }^{[11]}$.

Table IV Minimum and maximum values of heat flux and current density. 


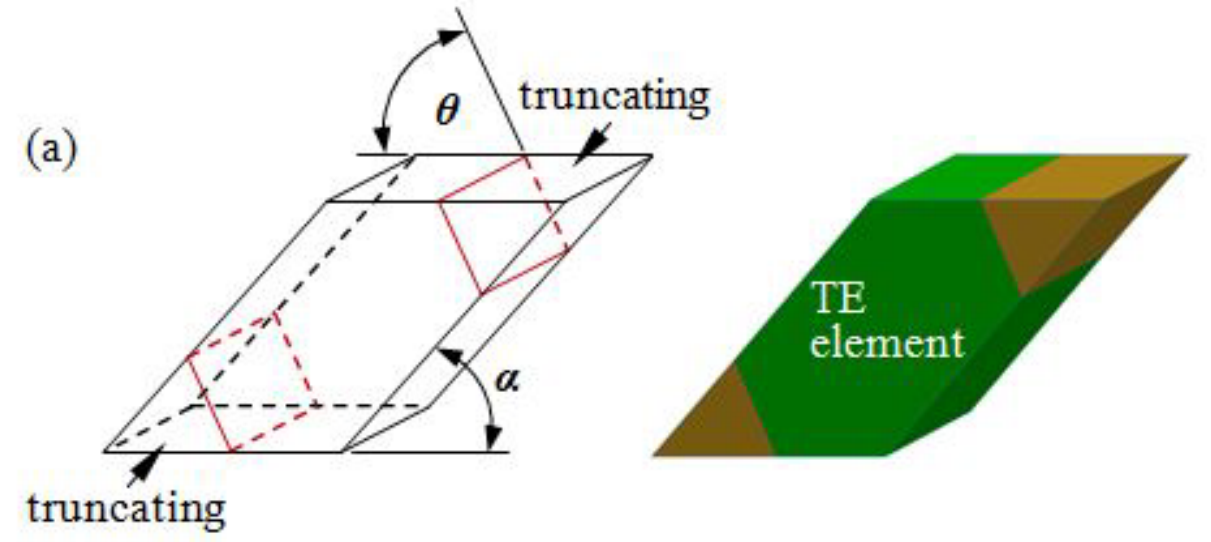




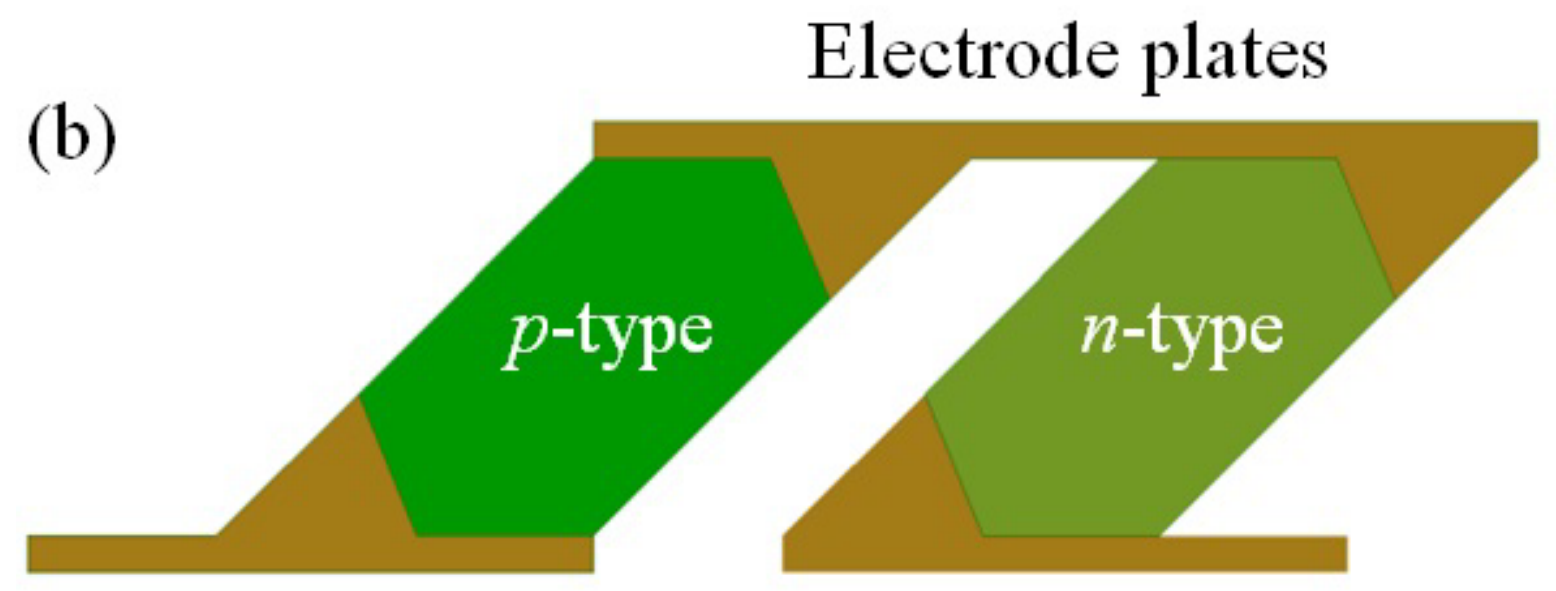




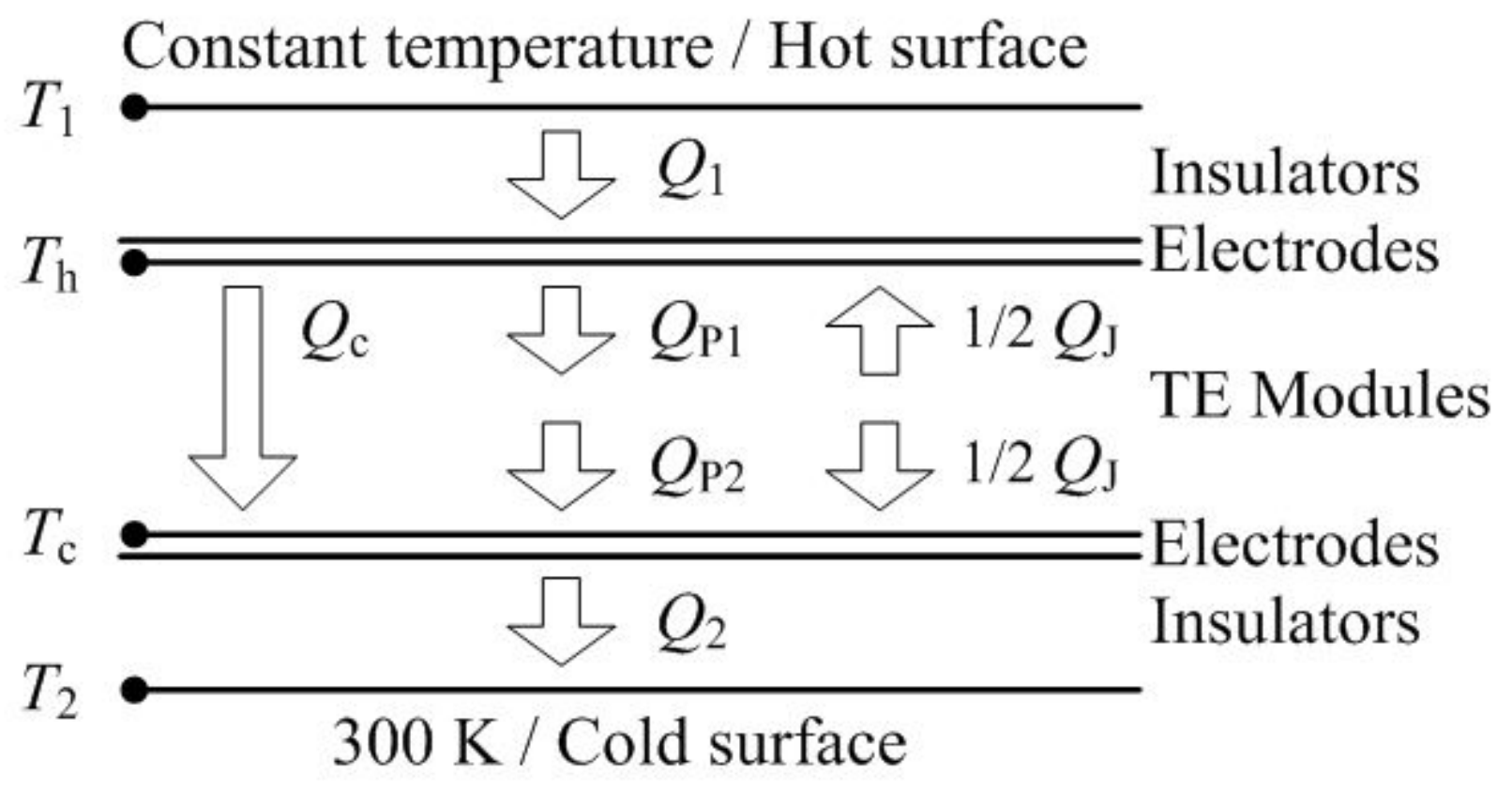




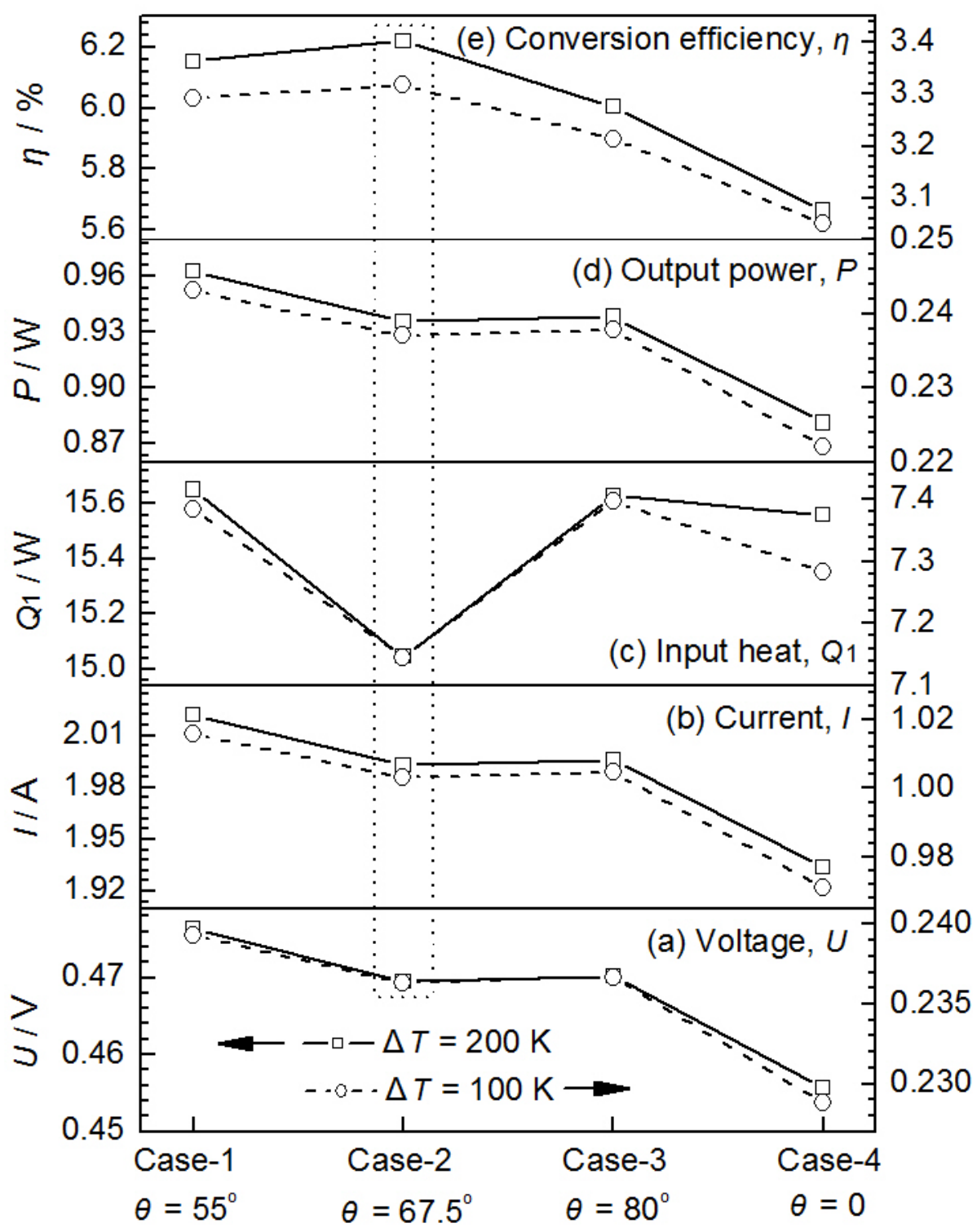



0.0
5.3
10.6
16.0
21.2

\section{(a) Case-2 (Vector $\left./ \mathrm{MA} \cdot \mathrm{m}^{-2}\right)$}

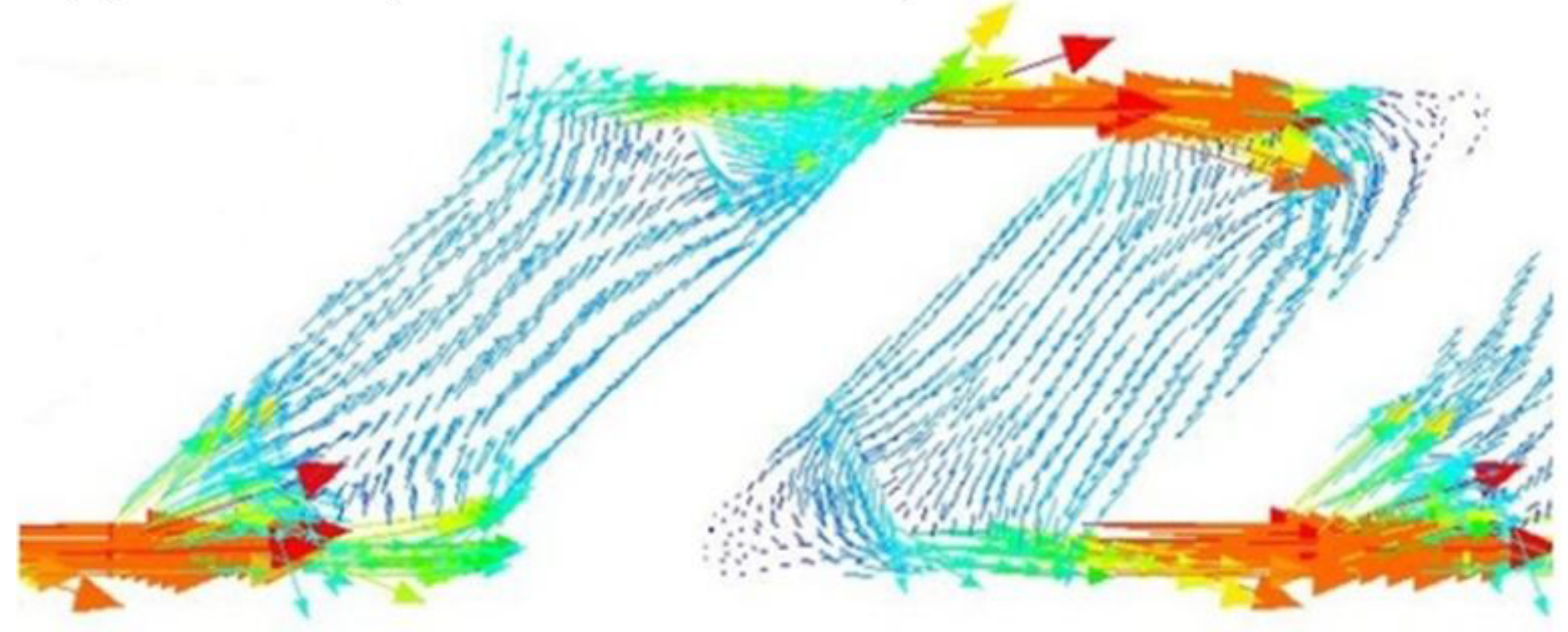



0.0
5.2
10.3
15.4
20.5

\section{(b) Case-4 (Vector $/ \mathrm{MA} \cdot \mathrm{m}^{-2}$ )}

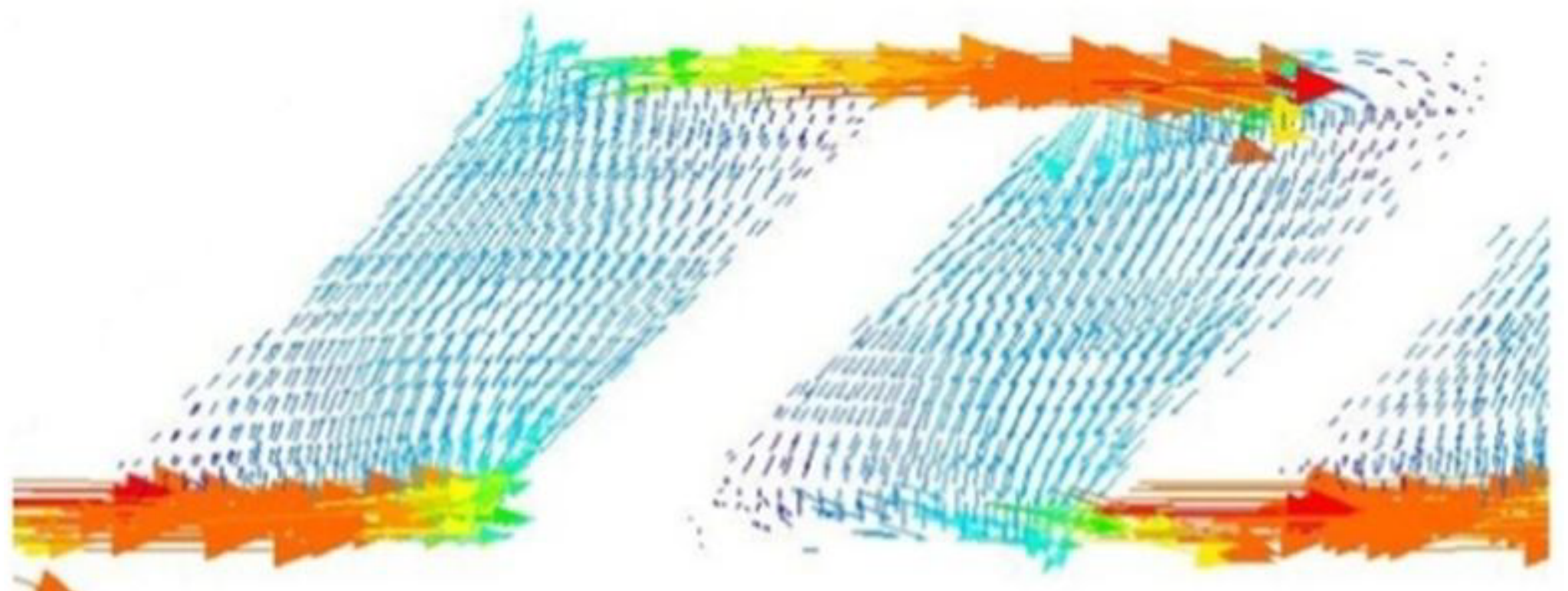




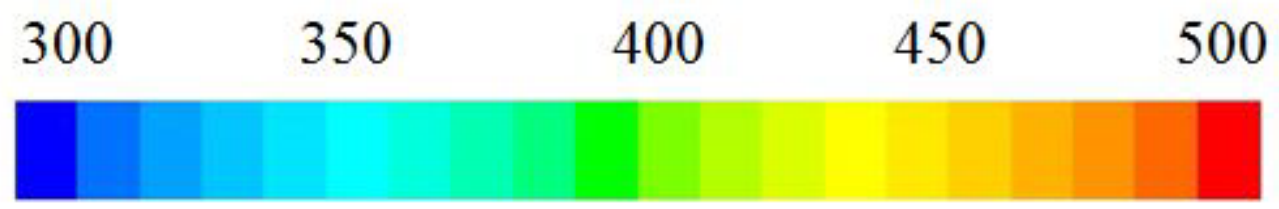

(a) Case-2 (Contour / K)

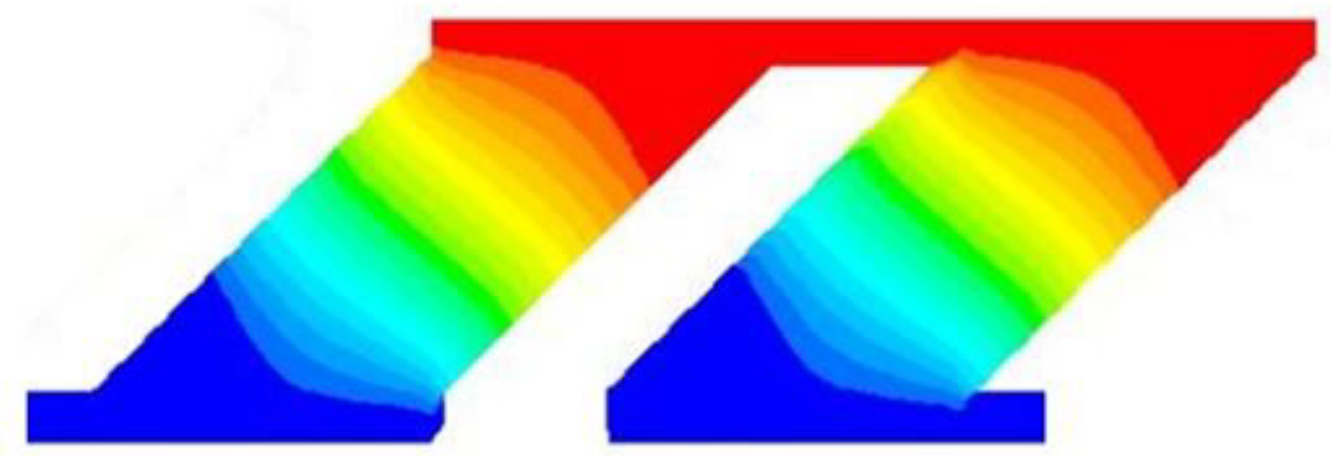




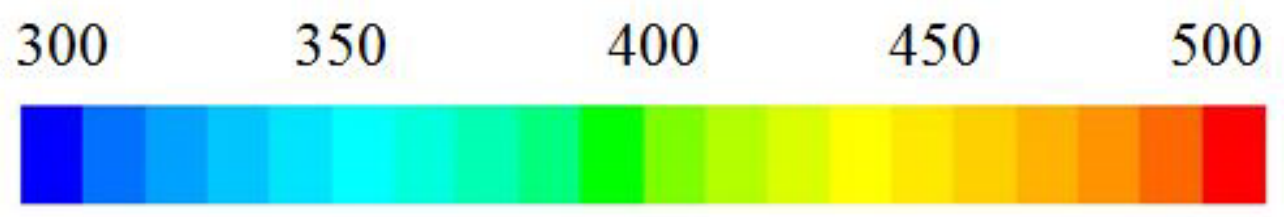

(b) Case-4 (Contour / K)

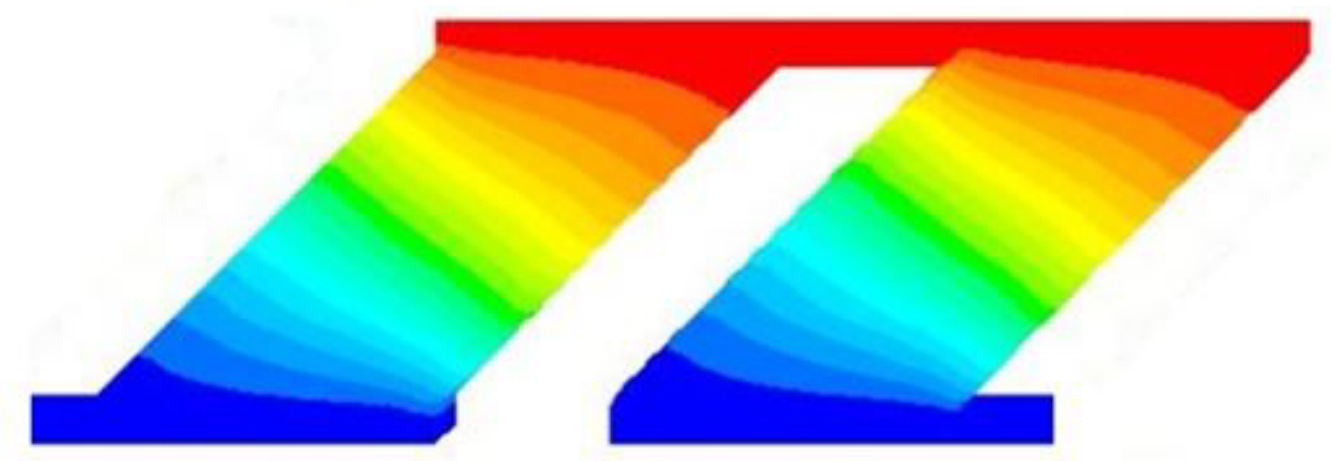




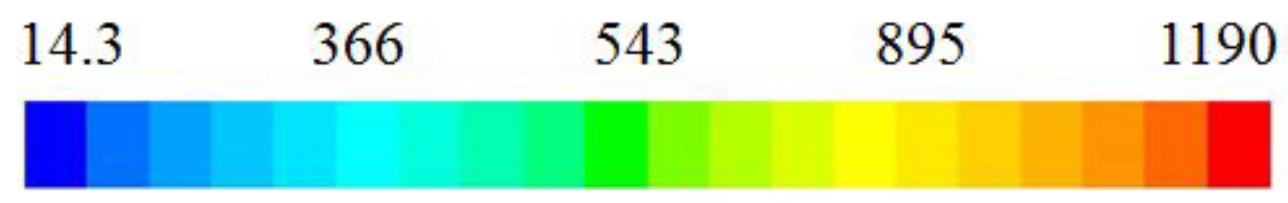

(a) Case-1 (Vector $/ \mathrm{kW} \cdot \mathrm{m}^{-2}$ )

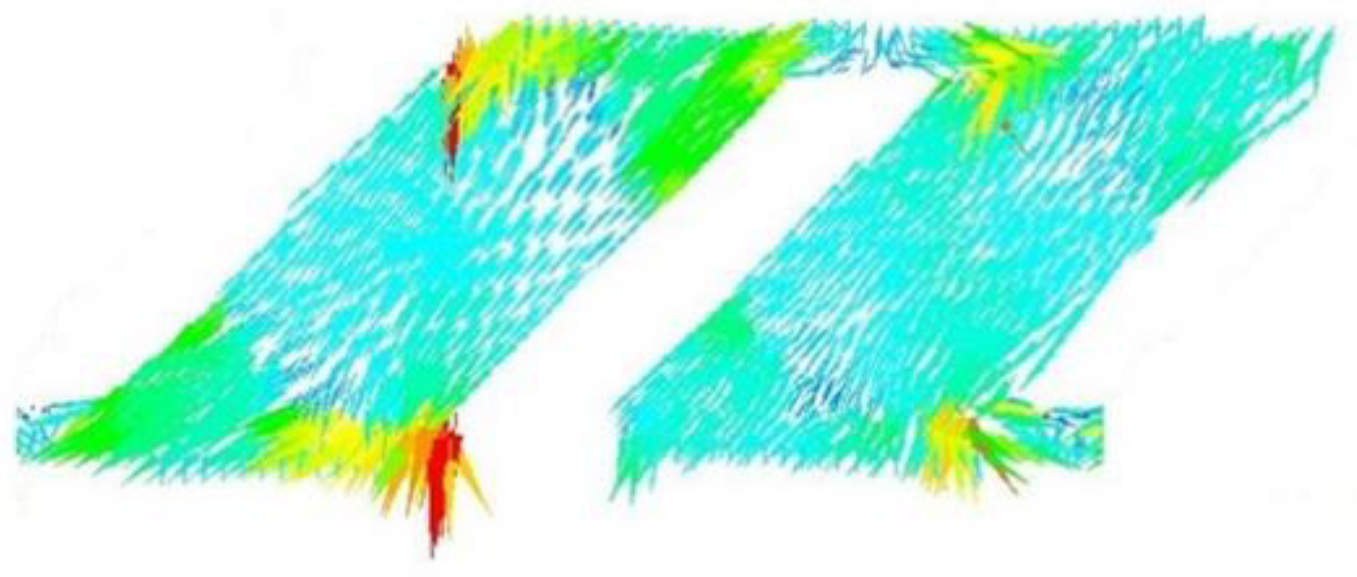




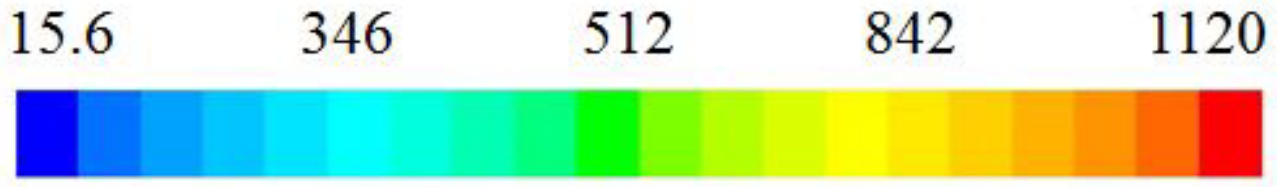

(b) Case-2 (Vector $/ \mathrm{kW} \cdot \mathrm{m}^{-2}$ )

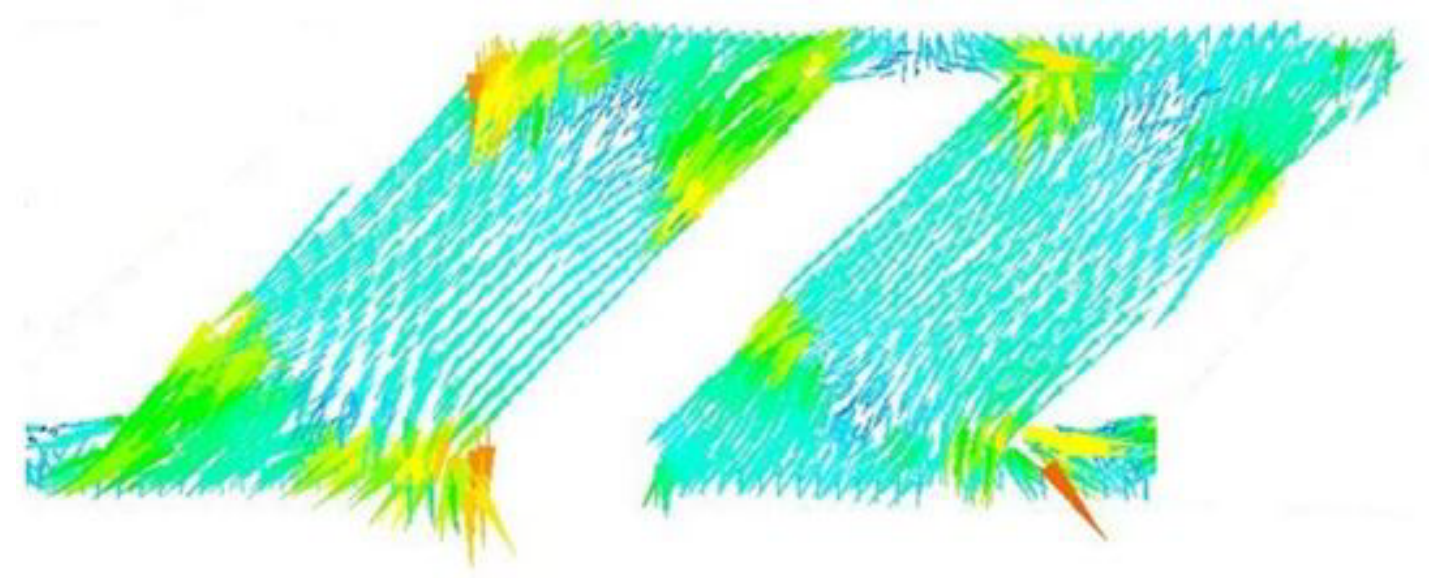




\begin{tabular}{lllll}
15.8 & 381 & 624 & 935 & 1230 \\
\hline
\end{tabular}

(c) Case-3 (Vector $/ \mathrm{kW} \cdot \mathrm{m}^{-2}$ )

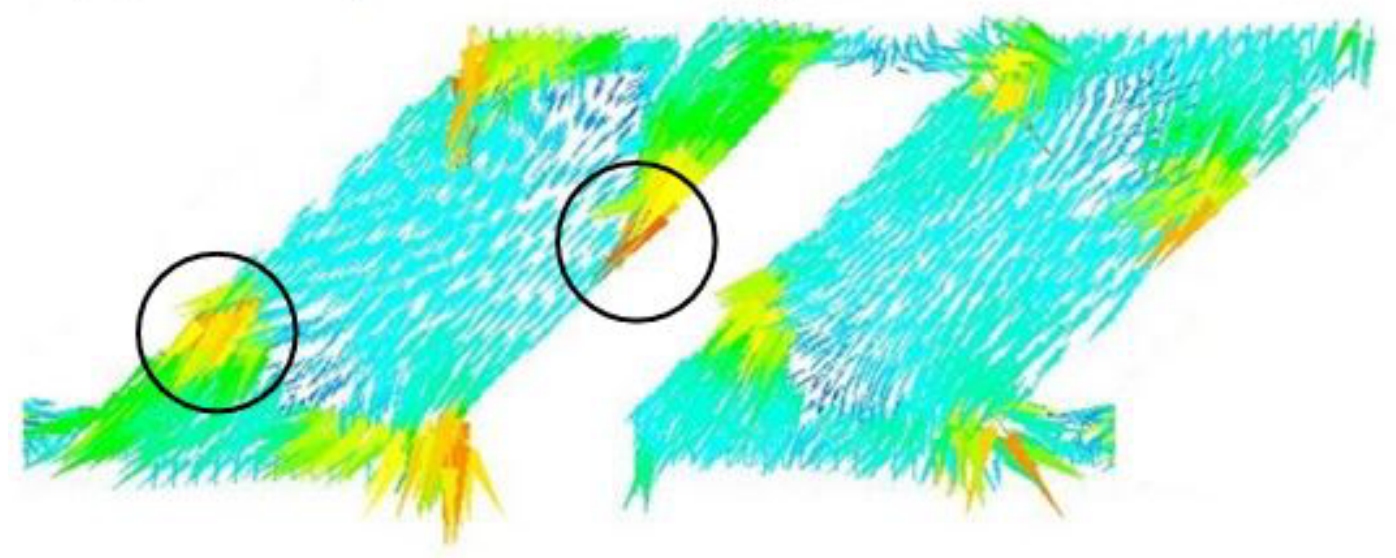




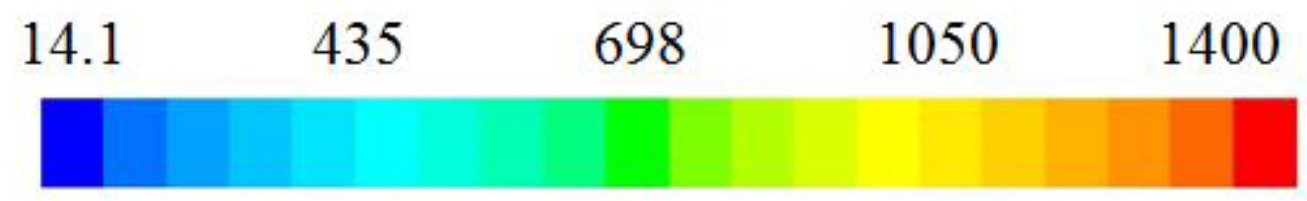

\section{(d) Case-4 (Vector $/ \mathrm{kW} \cdot \mathrm{m}^{-2}$ )}

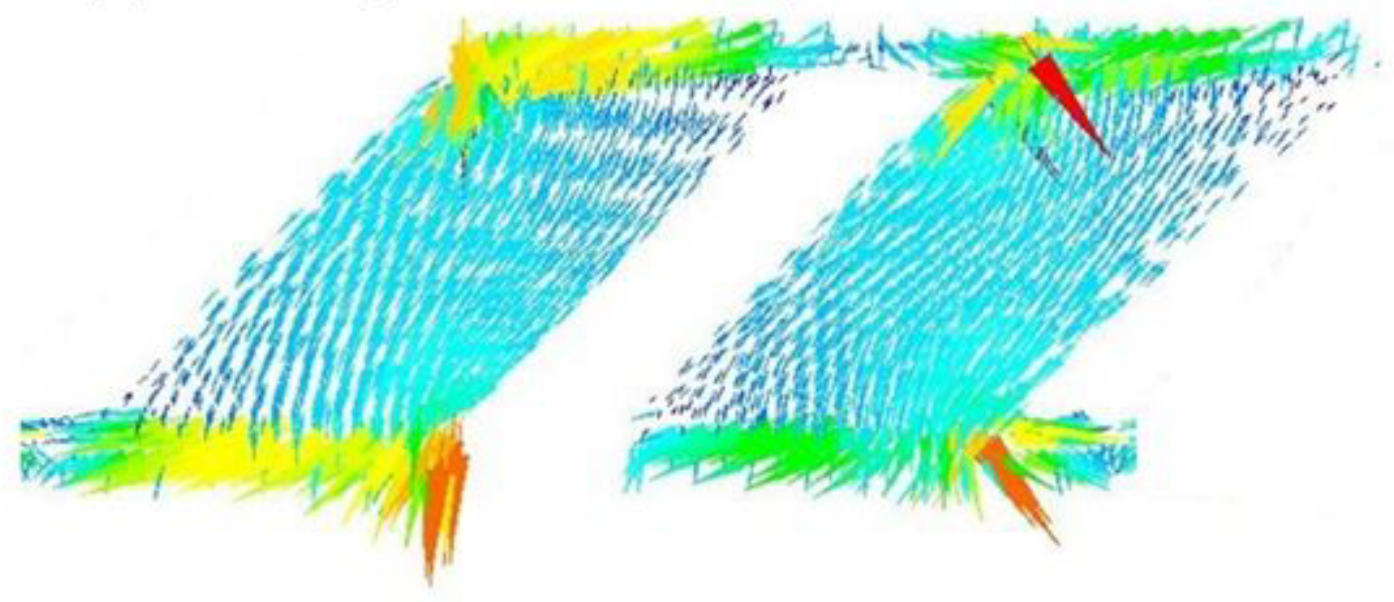


Table I Designs of tilted polyhedron elements.

\begin{tabular}{cc}
\hline Designs & Truncating angles, $\theta$ (degree) \\
\hline Case-1 & 55.0 \\
Case-2 & 67.5 \\
Case-3 & 80.0 \\
Case-4 & 0 \\
\hline
\end{tabular}


Table II Configuration data of TE module.

\begin{tabular}{|c|c|c|c|c|c|c|c|}
\hline \multirow[b]{2}{*}{$p-n$ pairs } & \multicolumn{3}{|c|}{ Elements ( $p$-type and $n$-type) } & \multicolumn{2}{|c|}{ Electrodes } & \multicolumn{2}{|c|}{ Insulators } \\
\hline & $\begin{array}{c}\text { Area } \\
\left(\mathrm{mm}^{2}\right)\end{array}$ & $\begin{array}{l}\text { Leg height } \\
\text { (mm) }\end{array}$ & $\begin{array}{l}\text { Width } \\
(\mathrm{mm})\end{array}$ & $\begin{array}{c}\text { Thickness } \\
\text { (mm) }\end{array}$ & $\begin{array}{l}\text { Width } \\
\text { (mm) }\end{array}$ & $\begin{array}{c}\text { Thickness } \\
\text { (mm) }\end{array}$ & $\begin{array}{l}\text { Width } \\
\text { (mm) }\end{array}$ \\
\hline 18 & 1.0 & 1.0 & 1.0 & 0.1 & 1.0 & 0.5 & 1.0 \\
\hline
\end{tabular}


Table III Thermal and electric properties of materials ${ }^{[11]}$.

\begin{tabular}{|c|c|c|c|c|c|c|c|}
\hline & & \multicolumn{2}{|c|}{$\begin{array}{c}\text { Seebeck coefficient } \\
\left(\mu \mathrm{V} \cdot \mathrm{K}^{-1}\right)\end{array}$} & $\begin{array}{l}\text { Thermal conductivity } \\
\left(\mathrm{W} \cdot \mathrm{m}^{-1} \cdot \mathrm{K}^{-1}\right)\end{array}$ & $\begin{array}{l}\text { Electric resistivity } \\
\qquad(\mu \Omega \cdot \mathrm{m})\end{array}$ & \multicolumn{2}{|c|}{$\begin{array}{c}\text { Figure-of-Merit } \\
(-)\end{array}$} \\
\hline $\mathrm{Bi}_{2} \mathrm{Te}_{3}$ & (p-type) & 190 & $(300-500 \mathrm{~K})$ & 2.06 & 5.5 & 0.90 & $(300-600 \mathrm{~K})$ \\
\hline $\mathrm{Bi}_{2} \mathrm{Te}_{3}$ & (n-type) & -210 & $(300-500 \mathrm{~K})$ & 2.02 & 10.0 & 0.92 & $(300-600 \mathrm{~K})$ \\
\hline $\mathrm{Cu}$ & (electrode) & 1.83 & $(300 \mathrm{~K})$ & 398 & 0.0155 & & \\
\hline $\mathrm{Al}_{2} \mathrm{O}_{3}$ & (insulator) & & & 36 & 0 & & \\
\hline
\end{tabular}


Table IV Minimum and maximum values of heat flux and current density.

\begin{tabular}{ccccccc}
\hline \multirow{2}{*}{ Designs } & \multicolumn{2}{c}{ Heat flux $\left(\mathrm{kW} \cdot \mathrm{m}^{-2}\right)$} & & \multicolumn{2}{c}{ Current density $\left(\mathrm{MA} \cdot \mathrm{m}^{-2}\right)$} \\
\cline { 2 - 3 } \cline { 6 - 7 } & Minimum & Maximum & & Minimum & Maximum \\
\hline Case-1 & 14.3 & 1190 & & 0 & 21.6 \\
Case-2 & 15.6 & 1120 & & 0 & 21.2 \\
Case-3 & 15.8 & 1230 & & 0 & 22.1 \\
Case-4 & 14.1 & 1400 & & 0 & 20.5 \\
\hline
\end{tabular}

